\section{Asociación Vecinal Villa del Parque de Santa Fe: el desafío hacia la autogestión en clave de Economía Social y Solidaria}

\author{
María Rut Azerrad \\ mazerrad@fce.unl.edu.ar \\ (iD) orcid.org/0000-0002-6888-5840 \\ Germán Rossler \\ rosslergermán@gmail.com \\ (iD) orcid.org/0000-0003-0129-6264 \\ Universidad Nacional de Litoral, Argentina
}

RECEPCIÓN: 26/06/20

ACEPTACIÓN FINAL: 04/09/20

\section{Resumen}

A partir del año 2011 un equipo

interdisciplinario de la Facultad de Ciencias

Económicas de la Universidad Nacional del

Litoral trabajó con la Asociación Vecinal Villa

del Parque de la ciudad de Santa Fe a través

de dos Proyectos de Extensión de Interés

Social con el objetivo de fortalecer la capacidad

de autogestión de su Comisión Directiva. Estos proyectos se articularon sobre dos líneas de acción: la primera relacionada con cuestiones contables, administrativas, legales, y con el proceso de toma de decisiones; la segunda vinculada al desarrollo de una estrategia de "obra-escuela" con una cooperativa de trabajo. Este artículo tiene como objetivo reflexionar sobre las etapas que atravesaron estos proyectos focalizándose en las relaciones entre los actores sociales participantes, los obstáculos que surgieron y las modificaciones realizadas en las estrategias para lograr un diálogo de saberes.

Palabras clave: extensión universitaria; Economía Social y Solidaria; asociación vecinal; autogestión.
Villa del Parque Neighborhood

Association from Santa Fe: the challenge towards self-management from Social and Solidarity Economy

\section{Abstract}

Since 2011, an interdisciplinary team from the Faculty of Economic Sciences of the Universidad Nacional del Litoral worked with the Villa del Parque Neighborhood Association of the city of Santa Fe through two Extension Projects of Social Interest (PEIS). With the aim of strengthening the self-management capacity of its Board of Directors, these projects were articulated on two lines of action: the first, related to accounting, administrative, legal issues and the decision-making process; the second linked to the development of a "workschool" strategy with a work cooperative. This article aims to reflect on the stages these projects went through, focusing on the relationships between the participating social actors, the obstacles that arose and the modifications made in the strategies to achieve a dialogue of knowledge.

Keywords: university extension; Social and Solidarity Economy; neighborhood association; self-management.
Sujetos y relaciones en extensión universitaria / Intervenciones 다(1)(2)(2)
Associação de moradores de Villa del Parque de Santa Fe: o desafio para a autogestão em chave da Economia Social e de Solidariedade

\section{Resumo}

A partir de 2011, uma equipe interdisciplinar da Faculdade de Ciências Econômicas da Universidad Nacional del Litoral trabalhou com a Associação de Moradores Villa del Parque da cidade de Santa Fé por meio de dois Projetos de Extensão de Interesse Social (PEIS). Com o objetivo de fortalecer a capacidade de autogestão do seu Conselho de Administração, esses projetos se articulam em duas linhas de atuação: a primeira, relacionada às questões contábeis, administrativas, jurídicas e ao processo decisório; a segunda vinculada ao desenvolvimento de uma estratégia "trabalhoescola" com uma cooperativa de trabalho. Este artigo tem como objetivo refletir sobre as etapas pelas quais esses projetos passaram, focalizando as relações entre os atores sociais participantes, os entraves que surgiram e as modificações realizadas nas estratégias para o diálogo de saberes.

Palavras-chave: extensão universitária; Economia Social e Solidária; associação de moradores; autogestão.

Para citación de este artículo: Azerrad, M. R. y Rossler, G. (2020). Asociación Vecinal Villa del Parque de Santa Fe: el desafío hacia la autogestión en clave de Economía Social y Solidaria. +E: Revista de Extensión Universitaria, 10(13), e0011. doi: 10.14409/extension.2020.13.Jul-Dic.e0011 


\section{Introducción}

La Facultad de Ciencias Económicas (FCE) de la Universidad Nacional del Litoral (UNL) trabajó con la Asociación Vecinal Villa del Parque de la ciudad de Santa Fe desde el año 2011 hasta 2018 a través de dos Proyectos de Extensión de Interés Social (PEIS). ${ }^{1}$ Su objetivo de contribuir al desarrollo institucional y la capacidad de autogestión de su Comisión Directiva constituyó un desafío académico.

Los proyectos se articularon en torno a dos líneas de acción: la primera de ellas relacionada con cuestiones contables, administrativas, legales, el fortalecimiento de toma de decisiones y la resolución de problemas; la segunda vinculada al desarrollo de una estrategia de "obra-escuela" junto con la Cooperativa de Trabajo Jóvenes de San Cayetano, trabajo que se desarrolló en la segunda etapa de los proyectos.

La finalidad de este escrito es reflexionar sobre las etapas que atravesaron los integrantes del equipo universitario y de la Asociación Vecinal durante estos proyectos haciendo énfasis en: a) los actores sociales participantes y las relaciones que se establecieron; b) obstáculos que surgieron en el fortalecimiento de las condiciones de autogestión; c) modificaciones en las estrategias que se planificaron para la construcción de un diálogo de saberes.

El artículo se encuentra estructurado de la siguiente manera: en la primera sección se presenta una breve descripción del barrio Villa del Parque como territorio de intervención, con sus trayectorias históricas e institucionales. Posteriormente se expone el marco epistemológico y teórico desde el cual se planificaron e implementaron las distintas intervenciones y acciones en el contexto de la Asociación Vecinal de Villa del Parque. Luego se identifican los obstáculos que surgieron al interior de su Comisión Directiva durante el proceso de construcción de su autogestión, considerando los cambios realizados en la estrategia de intervención y en la metodología de trabajo utilizada por el equipo extensionista para tratar de superarlos. Finalmente, se establecen los desafíos que presenta la construcción del proceso autogestivo en la Vecinal Villa del Parque, colocando el foco en su resignificación desde los espacios asociativos y cooperativos.

\section{Barrio Villa del Parque: organización popular, historia y presente}

En el presente apartado se realiza una breve caracterización del barrio Villa del Parque y su historia de organización popular con el propósito de comprender el contexto en el que se desarrolló la intervención social sobre su Asociación Vecinal. Para lograr esta caracterización se recurrió a la información recabada a partir de una serie de entrevistas semiestructuadas efectuadas previamente a la formulación de los proyectos de extensión con integrantes de la Comisión Directiva de la Asociación Vecinal. Por medio de este instrumento se accedió a la perspectiva de los actores sobre el espacio escénico: el territorio y su entramado culturalpolítico-ambiental (Carballeda, 2008).

1) PEIS: "Autogestión de la Vecinal Villa del Parque. Factores críticos para el desarrollo y fortalecimiento de las capacidades y saberes de los actores involucrados en el marco de la Economía Social y Solidaria", dirigido por la Mg. CPN María Rut Azerrad. Res. CS 394/14. PEIS: "Asociación Vecinal Villa del Parque: fortalecimiento para la autogestión en clave a Economía Social y Solidaria”, dirigido por la Mg. CPN María Rut Azerrad. Res. CS 665/16. 
Luego se hace un recorrido sobre sus legados institucionales, desde su fundación hasta la actualidad, enmarcando esta organización asociativa en el campo de la Economía Social y Solidaria (ESS).

El barrio Villa del Parque ${ }^{2}$ está ubicado en el distrito oeste de la ciudad de Santa Fe, y pertenecen a su jurisdicción alrededor de 3300 vecinos. Es uno de los más pequeños de la ciudad, con 36 manzanas aproximadamente, y limita con los barrios Barranquitas, Villa Oculta, Santa Rosa de Lima y Roma.

Villa del Parque comenzó a poblarse en las décadas del 30 y del 40 a partir del proceso de industrialización por sustitución de importaciones. Durante esas décadas se produjo el movimiento migratorio del campo a las grandes ciudades, donde fábricas y talleres comenzaban a incorporar trabajadores. Muchas familias del norte argentino se radicaron en el barrio, asentándose en casas precarias, levantadas con chapas y cartones.

La Asociación Vecinal de Villa del Parque se fundó el 27 de enero de 1955 con el objetivo de reclamar por un conjunto de carencias que existían en este barrio marginado de la ciudad de Santa Fe y luchar por la inclusión y la igualdad. Una figura clave en la organización popular del barrio fue el párroco Osvaldo Catena, ${ }^{3}$ quien desde 1956 y durante 20 años lideró un proceso importante de cambio en el barrio. En la década del 60 tuvo un papel central trabajando junto con la Vecinal en el proceso del loteo que permitió el acceso a la vivienda a muchas familias, el logro de servicios básicos y la creación de las primeras instituciones. Sin embargo, en el año 1976, con la llegada de la dictadura militar a la Argentina, este trabajo en el campo social se vio interrumpido abruptamente. ${ }^{4}$

Con el retorno a la democracia, la Vecinal volvió a abrir sus puertas pero funcionó bajo el control de grupos políticos partidarios, quienes no se rigieron por los Estatutos ni realizaron elecciones de autoridades. Luego, en el año 2003, la corrupción y el abandono estatal dieron lugar a los crímenes hídricos ${ }^{5}$ con la inundación más importante de la historia de nuestra ciudad, por la cual se produjo la muerte de numerosos vecinos del barrio. ${ }^{6}$ Finalmente, en 2008 el gobierno municipal intervino la Vecinal y se volvieron a realizar elecciones, en las cuales resultó ganadora una lista de vecinos autoconvocados que conformaron la Comisión Directiva, la que demandó la presencia y solicitó apoyo a la FCE de la UNL.

En la actualidad, esta Comisión Directiva representa la voz de los vecinos ante el gobierno local sobre los problemas que afectan al barrio caracterizado por un alto grado de vulnerabilidad socioeconómica. El entramado social presenta un notorio déficit de servicios públicos

2) El barrio Villa del Parque no siempre se denominó así. Primero se lo conoció como "el campo de Stardel”, después como el barrio "Los Bravos" (no porque hubiera gente conflictiva sino porque había una familia numerosa de apellido Bravo). Más adelante se lo llamó "El Triángulo", por la forma geográfica que le dan las vías férreas.

3) Enrolado en el Movimiento de los Sacerdotes para el Tercer Mundo, hizo que varios religiosos iniciaran un trabajo voluntario en Villa del Parque.

4) El padre Catena debió dejar el lugar tras recibir amenazas de muerte. Durante esos años la Vecinal dejó de funcionar debido al avance de la represión estatal.

5) Nos permitimos usar esta expresión tomando las palabras de los damnificados por la inundación de la ciudad de Santa Fe causada por el desborde del río Salado, que anegó un tercio de la ciudad y causó la muerte de 158 personas, para destacar la responsabilidad política de los gobiernos provincial y municipal de ese momento.

6) Susann Ullberg de Baez (2015) hace una lectura sobre la relación entre desastres, memoria y economía solidaria en el barrio Villa del Parque. 
(no hay red de gas natural, solo algunos sectores del barrio cuentan con asfalto, agua potable, desagües, cloacas, y no ingresa ninguna línea de colectivos).

María Ledesma — secretaria de la Asociación Vecinal y expresidenta de la misma— relaciona la ausencia de políticas públicas por parte del Estado con la identidad vecinalista de la nueva Comisión Directiva, que va más allá de cualquier identidad política partidaria. Sobre esta cuestión expresó:

"Tal vez porque no nos identificamos políticamente con ningún grupo partidario estamos en el olvido. Nuestra línea vecinalista es no embanderarnos partidariamente. Recibimos a todos, pero no nos identificamos con nadie. La Vecinal es la casa del vecino, los invitamos a todos a participar y a integrarla. También los convocamos a compartir las reuniones de Comisión Directiva porque es importante el punto de vista de todos, porque estamos trabajando por y para ellos". (Entrevista 1, 11 de noviembre de 2013)

En este fragmento se observa que surge la necesidad de buscar respuestas en la cercanía, en el barrio, junto a los vecinos, desde formas de organización más flexibles que los partidos políticos. Como sostiene Leandro López (2005):

"El militante deja paso al participante, la verticalidad a posibilidades de horizontalidad, la política como espacio solo vinculado al Estado, a la política, para la solución de los problemas de la vida cotidiana desde la cooperación diaria". (p. 5)

A partir de esta búsqueda de soluciones para los problemas de la vida cotidiana de los vecinos del barrio, la Asociación Vecinal trabaja de forma articulada mediante acuerdos con instituciones educativas y organizaciones no gubernamentales realizando distintas actividades y talleres.

Desde el año 2011 y hasta la actualidad se desarrollan talleres para chicos y para adultos, y funciona allí un Centro de Alfabetización y Educación Básica para jóvenes y adultos. A través de un proyecto de la UNL y del Sindicato de Luz y Fuerza se brinda un taller de lectura y narración para los chicos coordinado por la escritora santafesina Alicia Barberis.

También se articuló con el Colegio Nuestra Señora del Calvario, la Asociación Civil "Santa Fe en Actividad", la Asociación Generar y la Fundación EPyCA para lograr un Proyecto de Aprendizaje y Servicio por el que las alumnas de 4to. año de ese colegio hicieron diversas tareas en la Vecinal Villa del Parque.

Además, en 2011 comenzó a trabajarse con extensión universitaria de la UNL, lo cual permitió una serie de logros. En el marco de este trabajo se implementó un taller de concientización sobre alimentación sana que luego posibilitó generar espacios de panadería y repostería. Por otra parte, en la Vecinal funcionan consultorios jurídicos gratuitos gracias a un programa de extensión de la Facultad de Ciencias Jurídicas y Sociales (FCJS) de la UNL y la Municipalidad de Santa Fe. Y en la sede de la institución se presta ayuda en trámites de la Administración Nacional de la Seguridad Social (Anses) y ante la Defensoría del Pueblo.

De esta manera, la Asociación Vecinal promueve la mejora de las condiciones vida de los vecinos, reclama por los problemas del barrio frente a la ausencia de políticas públicas del Estado, y propone iniciativas sobre la provisión de servicios y ejecución de obras. También desarrolla una labor cultural por medio de la organización de cursos y talleres, y coopera con los organismos de asistencia social al facilitar el asesoramiento que se les requiera. 
Con el desarrollo de estas prácticas solidarias, centradas en la reproducción ampliada de la vida de los vecinos del barrio y la búsqueda del bien común, podemos sostener que la Asociación Vecinal Villa del Parque se inscribe dentro del amplio espacio de la ESS. En este sentido, cabe destacar que, como sostiene José Luis Coraggio (2016) desde el enfoque de la ESS:

"el sentido de la actividad económica está definido como la generación de las bases materiales, y de sus condiciones subjetivas asociadas, requeridas para sostener o lograr el objetivo de la reproducción de la vida en algún ámbito específico". (p. 7)

A partir de esta conceptualización, es posible considerar que la Asociación Vecinal desarrolla una actividad socioeconómica en tanto contribuye a la resolución directa e indirecta de necesidades complejas e integrales de sus asociados y de los vecinos del barrio. Por lo tanto, se considera a la Asociación Vecinal de Villa del Parque como un "Emprendimiento Asociativo no Mercantil" (EANM). Estos emprendimientos son definidos por José Luis Coraggio y Alberto F. Sabaté (2010) a partir de tres rasgos centrales:

"a) se trata de configuraciones humanas no compulsivas, b) que se sostienen sobre la base del trabajo, c) cuyo destino es la satisfacción de necesidades sociales de sus miembros y de comunidades más amplias". (p. 221)

Los EANM son aquellas organizaciones socioeconómicas que no producen para la venta ni cobran un precio por sus servicios pero admiten aportes de los asociados a un fondo común para cubrir gastos de funcionamiento. A este respecto, la Asociación Vecinal cuenta con alrededor de 160 socios que abonan una cuota mensual, y a este ingreso se suman el alquiler de las instalaciones (en especial de un Salón de Usos Múltiples - SUM-) para distintos eventos; los avisos publicitarios en el diario La Voz de Villa del Parque, ${ }^{7}$ y la gestión de algún subsidio estatal. Esto posibilita contar con recursos económicos para sostener el desarrollo de las distintas actividades mencionadas anteriormente y el mantenimiento de las instalaciones de la sede de la institución.

\section{Epistemologías del sur y ecología de saberes para la acción extensionista}

Para reflexionar desde la extensión universitaria sobre las relaciones entre los distintos actores sociales involucrados y las diversas modalidades de conocimiento en el trabajo en la Vecinal de Villa del Parque se propone partir de los lineamientos generales de las Epistemologías del Sur. Esta expresión elaborada por Boaventura de Sousa Santos (2009) puede ser definida como el conjunto de procedimientos, conceptos e ideas, con el propósito de validar, construir y reconstruir conocimiento mediante las luchas sociales que resisten al capitalismo, al colonialismo y al patriarcado.

Esta propuesta teórica se centra en los modos y en las condiciones de producción de conocimiento enunciados desde un Sur, refiriéndose metafóricamente a los lugares de ex-

7) Desde el año 2011 se ha recuperado La Voz de Villa del Parque, medio de expresión histórico de la vecinal cuyo lema es: "La voz que nunca calla ante las injusticias". Es una construcción colectiva de publicación mensual que expresa a cada una de las organizaciones y/o instituciones del barrio. Se publican 800 ejemplares de distribución gratuita. 
plotación y opresión donde se dan procesos de resistencia, en contraposición a un Norte entendido como el lugar del dominio. Según Sousa Santos (2011), las Epistemologías del Sur parten de tres premisas:

1) La comprensión del mundo es más amplia que la comprensión occidental del mundo, por lo que su transformación puede desarrollarse por otros modos impensables desde Occidente.

2) Existe una diversidad de formas de pensar, sentir, actuar, relacionarse entre seres humanos y vincularse con la naturaleza, distintas concepciones del tiempo, diferentes formas de organización política y económica.

3) Esta diversidad del mundo debe ser activada y transformada teórica y prácticamente desde la pluralidad de conocimientos, evitando su monopolización en una teoría general.

Desde la perspectiva de Sousa Santos, las Epistemologías del Sur parten de la idea de que cuando los oprimidos pasan del lugar de víctimas a resistentes se produce conocimiento que debe ser enunciado porque constituye un insumo fundamental para los procesos de emancipación social. En este sentido, las Epistemologías del Sur tienen como objetivo:

"identificar y valorizar lo que a menudo ni siquiera aparece como conocimiento a la luz de las epistemologías dominantes, lo que en su lugar surge como parte de las luchas de resistencia contra la opresión y contra el conocimiento que legitima esa opresión". (2018, p. 29)

Para lograr cumplir con este objetivo desarrollan dos procedimientos centrales: por un lado, la ecología de saberes, y por otro, la traducción intercultural.

Nos focalizaremos en la primera noción que consideramos central para la extensión universitaria. Se vincula con una ecología que parte del reconocimiento de la pluralidad de conocimientos y la interdependencia entre ellos sin afectar su autonomía. Según Sousa Santos (2011), la ecología de saberes:

"Consiste, por una parte, en explorar prácticas científicas alternativas que se han hecho visibles a través de las epistemologías plurales de las prácticas científicas y, por otra, en promover la interdependencia entre los conocimientos científicos y no científicos". (p. 36)

Se trata de la apuesta a la articulación y al diálogo entre las diferentes modalidades de conocimiento: ciencia moderna, saberes populares, ancestrales, etc. Y este diálogo se posibilita a partir de la comprensión del carácter incompleto de todos los conocimientos.

Así, con esta propuesta teórica de la ecología de saberes es posible resignificar las relaciones y los vínculos entre los conocimientos de distintos actores que participan de las prácticas de extensión. A partir del diálogo entre los conocimientos científicos y disciplinares producidos en la universidad, con los conocimientos y saberes cotidianos o populares, las prácticas ignorantes de otros modos diferentes son reconocidas y se transforman en prácticas de conocimiento de distintas modalidades, enriqueciéndose mutuamente mediante reconocimiento de su diversidad.

Estos desarrollos teóricos sobre ecología de saberes fueron incorporados a la estrategia metodológica en dos PEIS, sustentándose en el modelo sistematizado por el equipo de Atividades Curriculares de Integração Ensino, Pesquisa e Extensão (ACIEPEs) de la Universi- 
dade Federal de São Carlos (UFSCar), Brasil, desde la Incubadora Regional de Cooperativas Populares (INCOOP) ${ }^{8}$ (Azerrad, 2009). Este modelo se presenta en la Figura 1.

Figura 1: Estrategia general de intervención de la Incubadora Regional de Cooperativas Populares (INCOOP)

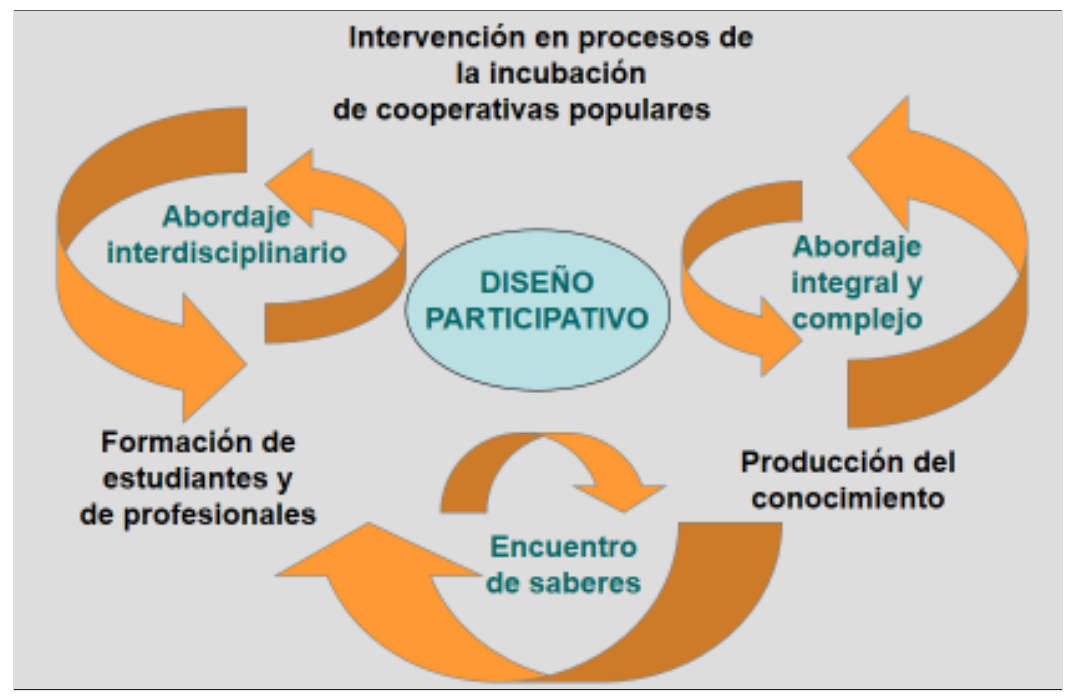

Fuente: Azerrad (2009).

Desde esta estrategia metodológica se promueve la integración de las tres funciones sustantivas de la universidad pública: docencia, investigación y extensión. Esta integración se hace visible entre la cátedra de Economía Social y Solidaria de la FCE — como espacio de formación de los estudiantes y de futuros profesionales-, la investigación a través de Cursos de Acción para la Investigación y Desarrollo $(\mathrm{CAl}+\mathrm{D})^{9}$ —con la finalidad de producir conocimiento y con la obtención de resultados válidos para el hacer científico en sí mismo y/o para aplicaciones de importancia económica, social o ambiental-, el Programa de Extensión de Economía Social y Solidaria y los proyectos de extensión - como modalidad de articulación con organizaciones sociales, iniciativas de ESS, distintos niveles gubernamentales y con la sociedad.

Esta estrategia de articulación de las tres funciones se estructura a partir del diseño participativo que implica una planificación situada, con una serie de acciones que contemple una mirada integral y compleja sobre la problemática identificada, recuperando los saberes de los actores sociales para la construcción conjunta de soluciones participativas en cada una de las etapas del proceso. Este tipo de diseño se contrapone con la metodología cerrada-secuencial, que se estructura en una temporalidad lineal y a partir de una reacción causa-efecto unidireccional.

8) Este abordaje metodológico es aprendido mediante la experiencia desarrollada por una de las autoras en una visita a la Universidad Federal de San Carlos en el Estado de San Pablo, Brasil, en el marco del Programa de Movilidad Escala Docente, articulado en el Comité Académico sobre Procesos Cooperativos e Iniciativas Económicas Asociativas (PROCOAS) de la Asociación de Universidades del Grupo Montevideo (AUGM).

9) Puntualmente, con el CAl+D 2016 "Red Colaborativa Solidaria de cooperativas de trabajo de la ciudad de Santa Fe. El rol articulador de Universidad Pública, sus alcances y limitaciones", dirigido por María Rut Azerrad. 


\section{¿Por qué fortalecer la autogestión de la Vecinal de Villa del Parque?}

Las luchas que se dan en diferentes ámbitos y contextos debido a la resistencia a las formas de dominación constituyen el elemento central a ser estudiado por las Epistemologías del Sur. Los casos con los que se trabaja desde el Programa de Extensión de Economía Social y Solidaria de la UNL, particularmente la experiencia de la Asociación Vecinal de Villa del Parque, son parte de esas luchas y de alternativas que desde el campo de la ESS enuncian otros modos de ser y estar en el mundo. Desde sus prácticas e iniciativas (los reclamos por los problemas del barrio para lograr más servicios y obras, las actividades culturales, y la articulación con otras instituciones de asistencia social) se intenta combatir y resistir a las distintas formas de desigualdades (sociales, económicas, políticas, etc.) a partir de relaciones basadas en la solidaridad.

Desde la perspectiva de Boaventura Sousa Santos y César Rodríguez (2007):

"Lo que se requiere es centrar la atención simultáneamente en la viabilidad y en el potencial emancipatorio de las múltiples alternativas que se vienen formulando y practicando alrededor del mundo que representan formas de organización económicas basadas en la igualdad, la solidaridad y la protección del medio ambiente." (p. 9)

De esta manera, centrándonos en la viabilidad y las condiciones de sostenibilidad de la experiencia de la Asociación Vecinal Villa del Parque, entendida como un espacio de ESS y una expresión de estas alternativas a la lógica de dominación hegemónica, nos propusimos fortalecer la autogestión de su Comisión Directiva.

En este sentido, siguiendo a Albuquerque (2004), desde los espacios asociativos y cooperativos la autogestión puede ser definida como:

"un sistema de organización de las actividades sociales, desarrolladas mediante la acción intencional y convergente de varias personas (actividades productivas, servicios, actividades administrativas), donde las decisiones relativas a los destinos del grupo son directamente tomadas por los participantes, con base en la atribución del poder decisorio a las colectividades definidas por cada una de las estructuras específicas de actividad (empresa, escuela, barrio, etc.)". (p. 44)

Respecto de la Asociación Vecinal, de acuerdo con su Estatuto, ${ }^{10}$ el poder decisorio es otorgado a través de una Asamblea General de socios a una Comisión Directiva que debe encargarse de dirigir y administrar la institución. Por este motivo se tomó como la población de referencia para el proceso de fortalecimiento de la autogestión.

Además, a partir de esta revisión teórica del concepto de autogestión es posible reflexionar: ¿por qué fortalecer la autogestión de la Asociación Vecinal Villa del Parque? ¿Por qué no simplemente hablar de gestión? Porque, como sostiene Albuquerque (2014), la autogestión en estos tipos de espacios asociativos:

"saca el concepto de gestión del dogmatismo, del carácter unilateral, de la esclerosis, del fanatismo, y del espíritu categórico, de los elementos de intimidación, de la ingenuidad y de las ilusiones de una fijación sobre un solo plano: del concepto de gestión apenas cómo un ejercicio técnico especializado". (p. 383) 
Esta concepción de autogestión que trata de evitar los rasgos dogmáticos y unilaterales favorece la construcción de una ecología de saberes. En primer lugar, al reconocer la validez de los conocimientos populares que los integrantes de la Comisión Directiva de la vecinal lograron aprender desde sus diversas experiencias educativas formales e informales y desde sus vidas cotidianas en el orden sociopolítico del barrio. En segundo lugar, al reconocer los límites del conocimiento académico y disciplinario, por lo que se hace necesaria la construcción de una perspectiva interdisciplinaria e integral a través de espacios de diálogo entre el mundo académico y la perspectiva de los actores sociales, sus expectativas, intereses, motivaciones y significados.

\section{La construcción de autogestión de la Comisión Directiva de la Asociación Vecinal Villa del Parque como proceso dinámico}

A continuación se narran las distintas etapas del proceso de construcción de autogestión de la Comisión Directiva de Villa del Parque a partir tres ejes de análisis:

a) Los actores sociales participantes y las relaciones que se establecieron.

b) Obstáculos que surgieron en el fortalecimiento de las condiciones de autogestión.

c) Modificaciones en las estrategias que se planificaron para la construcción un diálogo de saberes.

Desde el acercamiento de la Asociación Vecinal a la FCE de la UNL y durante el desarrollo de los dos PEIS podemos diferenciar tres etapas.

\section{Primera etapa}

En el año 2011, integrantes de la Comisión Directiva de la Vecinal se acercaron a la FCE de la UNL y solicitaron asesoramiento para realizar el balance de su institución. Desde ese momento se comenzó a trabajar desde el Programa de Economía Social y Solidaria realizando distintas actividades de asesoramiento y colaboración.

En esta primera aproximación, los integrantes de la Comisión Directiva tenían una necesidad cuya resolución requería un conocimiento disciplinario y un profesional que certificara el balance contable. Una vez resuelta esa necesidad, comenzó a gestarse un vínculo de confianza entre los integrantes de la Comisión Directiva y los representantes de la FCE a partir de este vínculo fundante para el diálogo y respeto mutuo, sustentado en la definición clara del alcance de nuestras tareas para definir el rol de la Universidad en una dimensión académica, despojada de asistencialismo y filantropía. Simultáneamente, se trabajó en establecer un vínculo de reciprocidad rescatando los saberes instalados y los aprendizajes comunes. Se desarrolló un proceso de involucramiento sobre el contexto social del barrio, su historia, sus características sociodemográficas, y las múltiples necesidades que presentaba la Comisión Directiva. Este proceso de aprendizaje complejo e integral y el diagnóstico inicial sirvieron como insumo estratégico para la segunda etapa.

\section{Segunda etapa}

En 2014 las acciones que se venían realizando en la Vecinal de forma aislada y fragmentada se articularon en el marco del PEIS y el voluntariado titulado "Autogestión de la Asociación Veci- 
nal Villa del Parque. Factores críticos para el desarrollo y fortalecimiento de las capacidades y saberes de los actores sociales involucrados en el marco de Economía Social y Solidaria". ${ }^{11}$

Desde este Proyecto de Extensión se identificaron en forma conjunta con los integrantes de la Comisión Directiva de la Vecinal las siguientes de problemáticas colectivas:

- Necesidad de acceder a capacitación técnica sobre gestiones administrativas y de control para la Comisión Directiva de la Vecinal.

- Asesoramiento continuo en la toma de decisiones relacionadas con las dinámicas de las demandas planteadas a la Comisión Directiva por parte de los asociados y vecinos.

- Realización de mejoras edilicias y de infraestructura para su normal funcionamiento ante la falta de financiamiento y de capacitación técnica.

- Alto nivel de desocupación de los vecinos del barrio.

A partir de estas problemáticas se determinaron los objetivos pertinentes:

- Contribuir al fortalecimiento institucional y a la capacidad de gestión y su articulación con el entramado social.

- Asistir el proceso de autonomía, aprendizaje y autogestión mediante la organización de espacios de capacitación, asesoramiento y cooperación que fortifiquen la capacidad de gestión de esta Asociación.

- Formar y consolidar una masa crítica de especialistas en el ámbito de la UNL desde un abordaje interdisciplinario.

En función de estos objetivos el proyecto se articuló en torno a dos líneas de acción: 1) la primera vinculada al asesoramiento en cuestiones contables, administrativas, legales; 2) la segunda atinente al desarrollo de una estrategia de "obra-escuela" junto con la Cooperativa de Trabajo Jóvenes de San Cayetano.

Primera línea de acción: fortalecimiento institucional

Respecto de la primera línea, a partir de un equipo interdisciplinario compuesto por estudiantes de distintas carreras, se realizaron distintas tareas vinculadas sobre todo a la incumbencia profesional de las ciencias económicas, contables y administrativas. Durante estas acciones se establecieron relaciones con otros actores, tanto del sector público como del privado, que implicaron distintas estrategias de acompañamiento y asesoramiento por parte del equipo y la puesta en práctica de múltiples conocimientos y saberes. A continuación se destacan las más relevantes:

- Se asesoró y acompañó a los integrantes de la Comisión Directiva en la gestión para la apertura de una cuenta corriente en una entidad bancaria. Para esto fue necesario reunir y organizar toda la documentación solicitada y cumplir con los requisitos establecidos por la entidad. La intervención de los integrantes del equipo fue estratégica para lograr la atención de los funcionarios y empleados de la institución bancaria que en primer término demostraron poco interés por solucionar el pedido directo del presidente y tesorero de la Asociación Vecinal. Al respecto, es posible destacar el rol facilitador que genera el respaldo institucional 
(a través del PEIS) de la UNL en el posicionamiento de la Vecinal frente a organismos externos tales como AFIP, instrucciones bancarias y otros organismos gubernamentales.

- Vinculado con esta cuestión, se confeccionó junto con el tesorero de la Vecinal una rendición de un subsidio del Ministerio de Desarrollo Social de la provincia para la construcción de un playón deportivo. Durante este proceso se manifestó la incapacidad de los procesos administrativos de los organismos municipales y provinciales para desarrollar normativas accesibles a los usuarios, junto con una ausencia plena de capacitación y acompañamiento para satisfacer las exigencias que se implementan. Luego de la entrega de la rendición los funcionarios públicos realizaron un control con inspecciones ex-post con una actitud examinadora, sin instrumentar evaluaciones para conocer el proceso.

- Para mantener el orden en la registración de operaciones contables, un insumo fundamental en la elaboración de las rendiciones de cuentas para el cierre del ejercicio, el equipo diseñó planillas de Egresos e Ingresos y posteriormente capacitó al tesorero en su utilización.

- En un trabajo articulado con la cátedra optativa y electiva de Economía Social y Solidaria de la FCE, un grupo de estudiantes y los integrantes de la Comisión Directiva realizaron los inventarios (el físico y el valorizado) para incorporar a la información de los Estados de Situación Patrimonial al cierre del Ejercicio Económico.

- Los integrantes del equipo del Proyecto confeccionaron los documentos de trabajo para los Balances Anuales Contables que incluyeron el Estado de Situación Patrimonial, Estado de Resultados y de Evolución del Patrimonio Neto, junto a los Anexos y las Memorias. Los mismos fueron presentados en las Asambleas Generales Ordinarias y miembros del PEIS acompañaron a la Comisión Directiva para facilitar su comprensión por parte de los asociados que participaron de estos eventos.

Es importante destacar el rol de la intervención del equipo del PEIS en la articulación con los profesionales que certificaron y firmaron los balances. En este sentido, la responsabilidad, compromiso y eficiencia para la confección de los mismos estuvo a cargo del equipo de trabajo de la facultad para luego acceder a la certificación final de los profesionales en un círculo virtuoso ganar-ganar. Estos profesionales aceptaron acompañar el trabajo del equipo entendiendo que se cubrían las garantías necesarias para respaldar con la acreditación y certificación de su firma.

Finalmente, con el asesoramiento y acompañamiento de estudiantes de abogacía de la FCJS de la UNL que efectuaron sus prácticas profesionales supervisadas en el marco del PEIS se procedió a la reforma del Estatuto Social de la Vecinal. En este proceso se consideró el Estatuto fundacional del año 1956 y su posterior reforma de 1976. Además, se utilizó como insumo el modelo de Estatuto elaborado por la Inspección General de Personas Jurídicas (IGPJ) de la Provincia de Santa Fe, acorde al nuevo Código Civil y Comercial de la Nación.

La reforma del cuerpo normativo interno que rige la vida de la Vecinal fue fundamental debido a que el manuscrito original era poco legible debido a su antigüedad y su posterior reforma no estaba integrada al cuerpo del mismo. De este modo se concretaron distintas reuniones entre los estudiantes y la Comisión Directiva en la sede de la Vecinal donde se discutieron por cada artículo las reformas necesarias para adecuar el Estatuto frente a las necesidades del nuevo contexto sociopolítico y considerando a las futuras generaciones de vecinalitas. Con posterioridad, la reforma fue aprobada en Asamblea General Extraordinaria y se logró que la misma fuera acreditada por la IGPJ. 
Por un lado, en el desarrollo de estas tareas y acciones los integrantes del equipo del PEIS y estudiantes pudieron conocer el proceso al interior de la Vecinal, sus fortalezas, sus debilidades y necesidades. Por otro lado, asimilaron los contenidos teóricos con las prácticas sociales y lograron una armónica utilización de conocimientos técnicos específicos de las diferentes disciplinas que interactúan en los grupos. Los estudiantes pudieron intervenir de manera profesional utilizando las herramientas conceptuales de acuerdo con su formación específica.

Sin embargo, los estudiantes también se enfrentaron a múltiples situaciones que no podían ser solucionadas desde estos conocimientos, debieron enfrentar la incertidumbre de no saber cuál era "la respuesta correcta" y atravesar la experiencia de construir, desde esta carencia de conocimiento, una posible respuesta en conjunto con los integrantes de la Comisión Directiva de la Vecinal, reivindicando los saberes y conocimientos desde una escucha y una interpelación mutua.

En tanto, los miembros de la Comisión Directiva pudieron reconocer a la Universidad como un actor clave para su asesoramiento y acompañamiento en la resolución de sus problemáticas. En especial, tuvieron la oportunidad de reconocer sus saberes y experiencias frente a los límites externos e internos del conocimiento académico en el proceso de coproducción de herramientas y soluciones para el fortalecimiento de la autogestión de la Vecinal.

También durante el desarrollo de estas actividades se presentaron ciertas dificultades al interior de la Comisión Directiva. Primero, la división de tareas entre sus integrantes se fue configurando de tal manera que algunos concentraron la mayoría de las tareas administrativas y en repetidas ocasiones no las pudieron cumplir en tiempo y forma. Esto generó tensiones con el resto de los integrantes y surgieron algunas rispideces en las relaciones interpersonales de la Comisión Directiva. Destacamos la incompatibilidad de intereses y la debilidad para identificar el beneficio colectivo por sobre las aspiraciones personales. Si bien no todos podían dedicarle el mismo tiempo a la Asociación Vecinal debido a que cada uno tenían ocupaciones personales diferentes (sus familias, sus trabajos, sus estudios, etc.), la dinámica de trabajo en equipo no lograba ser apropiada por parte de sus integrantes.

Luego, en el proceso de toma de decisiones y resolución de problemas, en el cual la Comisión Directiva contó con el asesoramiento y acompañamiento de los miembros del PEIS, hubo dificultades para diferenciar claramente el rol y el sentido de cada uno de los actores. Por esta razón en repetidas ocasiones el incumplimiento de las responsabilidades de algún integrante de la Comisión Directiva se trasladó a los miembros del PEIS.

Además, respecto de la asignación de responsabilidades igualmente hubo dificultades de comunicación entre el equipo del PEIS y la Comisión Directiva debido a que esta se canalizó generalmente a través de uno de sus miembros y de modo informal, lo cual impidió que los contenidos de la información se sociabilizaran con toda la Comisión Directiva y que sus integrantes dispusieran de la misma.

Ante estas tres dificultades, desde el equipo del PEIS se modificó la modalidad de trabajo al interior del mismo equipo ajustando los mecanismos de comunicación interno y reconociendo los desvíos en el alcance de la propia capacidad de gestión y las limitaciones de los objetivos planteados. Se autoevaluó y se reconoció que a veces resulta inútil hacer eficientemente la tarea equivocada. Estas modificaciones y ajustes se describirán al momento de presentar la tercera etapa del proceso de construcción de autogestión. 
Segunda línea de acción: obra-escuela

La segunda línea de acción del PEIS refiere al desarrollo de una estrategia de "obra-escuela" ${ }^{\prime 12}$ de capacitación en técnicas constructivas junto con la Cooperativa de Trabajo Jóvenes de San Cayetano en las instalaciones de la Vecinal. El propósito fue establecer vínculos por parte de la Comisión Directiva con otras organizaciones del barrio Villa del Parque para fortalecer el entramado y red conforme a los principios de la ESS. En este sentido, la Vecinal necesitaba realizar reformas edilicias para colocar un portón de entrada en su SUM, donde funciona un Centro de Alfabetización y Educación Básica para jóvenes y adultos, y se acordó contratar a la Cooperativa de Trabajo para realizar esta obra.

De esta forma, se trató de trabajar en un nivel mesoeconómico, definido por José Luis Coraggio (2016) como:

"conjuntos heterogéneos con potencial de complementariedad y generación de sinergias (usualmente) territoriales de relaciones intersubjetivas solidarias cotidianas de reciprocidad, en que se dan asociaciones libres y prácticas complementarias en base a la cooperación —no necesariamente formal— para la resolución de necesidades compartidas, lazos derivados de diversas relaciones de afinidad (vecindad, historia de asentamiento, identidad y cultura, comportamientos colectivos de representación o decisión y reglas comunes de resolución de conflictos, mutuo reconocimientos como actores locales, habitus, etc.)". (p. 8)

Con el propósito inicial de establecer un ámbito de confianza entre los integrantes de la Asociación Vecinal Villa del Parque y de la Cooperativa de Trabajo Jóvenes de San Cayetano, cuyos socios fundadores pertenecen al barrio de Villa del Parque, se desarrolló el "1er. Encuentro Vecinalista de Economía Solidaria". Este encuentro para generar lazos de vinculación y visibilidad de los referentes barriales de la Cooperativa con la Asociación Vecinal, y con especial énfasis con la Comisión Directiva, se diseñó en formato taller en el salón de reuniones de la Asociación Vecinal. Se trabajó fundamentalmente desde la complejidad de vinculaciones con el entorno, que incluye el barrio, articulando lazos sociales de reciprocidad, del desarrollo de valores e instituciones, y la necesidad de sostenibilidad de las políticas públicas redistributivas. Sin embargo, este propósito fue grata e inesperadamente superado por la participación espontánea de otros actores de la Economía Solidaria: integrantes de cooperativas de trabajo, de limpieza, de construcción, de servicios, de recicladores, y de empresas recuperadas de nuestra ciudad que excedieron los límites geográficos del barrio. Se identificó el valor de la solidaridad como el denominador común de las distintas organizaciones presentes y la necesidad de su práctica diaria. Asimismo, se logró generar un espacio de aprendizaje colectivo para la alfabetización en principios, valores y prácticas de ESS, tanto internamente para el equipo de trabajo de la universidad (lo que se manifestó desde el diseño previo del encuentro) como para los actores sociales de las distintas organizaciones participantes.

12) Los requisitos de la convocatoria para la aplicación de los fondos del proyecto impedían la compra directa de materiales o insumos para la mejora de las instalaciones. Sin embargo, autorizaban la compra de insumos para capacitación. Para respetar estas exigencias, se alinearon con una propuesta de capacitación a los jóvenes del barrio en técnicas constructivas en la sede de la Vecinal y se atendió la necesidad urgente de la mejora edilicia con financiamiento del proyecto. 
Dada la importante convocatoria que tuvo el primer encuentro se decidió realizar el "2do. Encuentro Cooperativista de Economía Solidaria" en la sede de la Cooperativa Jóvenes de San Cayetano. Del mismo formaron parte nuevas cooperativas y organizaciones sociales que plantearon la necesidad de comenzar a trabajar en red, articulando a nivel local. Esto luego posibilitó la elaboración de un Curso de Acción para la Investigación y Desarrollo (CAl+D) 2016, titulado "Red Colaborativa Solidaria de cooperativas de trabajo de la ciudad de Santa Fe. El rol articulador de la Universidad Pública, sus alcances y limitaciones". ${ }^{13}$

Respecto del proyecto de "obra escuela" la Cooperativa Jóvenes de San Cayetano realizó las reformas edilicias necesarias para su concreción, colocando un portón de entrada en el salón de la Asociación Vecinal. Sin embargo, la cooperativa tuvo dificultades para realizar adecuadamente la terminación de la obra, por lo cual el vínculo de confianza entre las dos instituciones establecido a partir de los encuentros previos se debilitó.

Esta dificultad llevó a pensar al equipo de trabajo del PEIS la necesidad de trabajar en el nivel microeconómico, limitando el alcance del proyecto al fortalecimiento institucional de la Comisión Directiva de la Vecinal de Villa del Parque. Esta modificación en el nivel de intervención se describirá en el siguiente apartado donde se caracteriza la tercera etapa por la que transcurrió el proyecto.

\section{Tercera etapa}

En función de las actividades desarrolladas se estableció un sólido vínculo de confianza entre los miembros de la Comisión Directiva y el equipo de trabajo de la UNL, por lo que se decidió dar continuidad al PEIS presentándose en la convocatoria para el año 2017. De esta forma, se trató de seguir consolidando el proceso de autogestión y realizando cambios en las estrategias de intervención y en la metodología de trabajo utilizada por el equipo extensionista para superar los obstáculos y dificultades que habían surgido.

Como mencionamos, se decidió trabajar en el nivel microeconómico, en donde según Coraggio (2016):

"se actúa sobre organizaciones económicas individuales (...) cuyos integrantes cooperan funcionalmente y pueden ser solidarios compartiendo su reproducción como objetivo común, o que, no siéndolo en el punto de partida, tienen el potencial para serlo". (p. 7)

Consideramos que al trabajar en este nivel, focalizándonos en la Comisión Directiva de la Vecinal, profundizamos y sostenemos los logros. Esta tercera etapa nos encontró, al equipo de trabajo, con el entrenamiento necesario para reconocer las exigencias y evitar desbordarnos por las necesidades genuinas y presentes en un contexto social de extrema vulnerabilidad. Reconocer el rol de la universidad pública es reconocer la capacidad de indignación por las desigualdades e injusticias y las limitaciones para resolverlas.

El recorte del alcance del proyecto sin dudas fue el resultado de la maduración del equipo de extensionista con el reconocimiento de la necesidad de transparentar los límites de las expectativas tanto internas como externas al proyecto. Además, como sostiene Coraggio (2016), la concreción de acciones eficaces a nivel maso requieren previamente de acciones

13) Es posible sostener que la universidad se conforma como un importante actor en el terreno de la ESS desde su rol articulador al estimular la organización de encuentros y visibilización de las y los actores de ESS en diferentes ámbitos. 
de nivel micro y esto se ve claramente en las dificultades que surgieron al intentar articular la Vecinal de Villa del Parque con la Cooperativa Jóvenes de San Cayetano.

Luego de redefinir el nivel de intervención se establecieron los siguientes objetivos:

1. Afianzar el proceso y desarrollo de la Asociación Vecinal Villa del Parque conforme a los principios y valores de la ESS.

2. Identificar los principales obstáculos que impiden el desarrollo de la autogestión de la Comisión Directiva de la Asociación Vecinal Villa del Parque y sus asociados.

3. Elaborar un plan de desarrollo institucional para la Asociación Vecinal Villa del Parque con estrategias que contemplen el corto, mediano y largo plazo.

4. Implementar capacitaciones, asesorías y asistencias técnicas desde una perspectiva integral (aspectos organizativos —-modelos de gestión-; contables y financieros; jurídicos y legales).

5. Generar instancias y prácticas de cooperación entre los asociados de la vecinal que faciliten y consoliden la creación de canales y formas de ayuda solidarias.

Para poder lograr estos objetivos se realizaron modificaciones en la metodología de trabajo. En primer lugar, se reestructuró la división de tareas entre los integrantes del PEIS de acuerdo con sus disciplinas e intereses. En segundo lugar, se decidió que los canales de comunicación fueran las reuniones de Comisión Directiva; con esta finalidad, dos integrantes del PEIS asistían a las mismas cada quince días para realizar el seguimiento de las actividades en conjunto con el Proyecto. Además, se creó un correo electrónico institucional del PEIS y se estableció que cualquier necesidad fuera transmitida por este medio.

En tercer lugar, se comenzaron a realizar informes de las reuniones de Comisión Directiva a las que se asistía. Estos fueron sistematizados en un cuadro de seguimiento de actividades con la finalidad de reflexionar en conjunto con la Comisión Directiva sobre cómo evolucionaba cada una de las actividades y si se cumplían en tiempo y forma para facilitar la necesidad de reconocer el compromiso implícito de ser parte de la Comisión.

En cuarto lugar, aprovechando la renovación de autoridades, desde el PEIS se propuso a los nuevos integrantes de la Comisión comenzar a trabajar aunadamente la organización de sus reuniones para mejorar su desarrollo. En este sentido, sugerimos que, al comienzo de las mismas, entre los presentes se consensuara un posible temario y se ordenaran los temas de acuerdo con su importancia. También destacamos la necesidad de que uno de los integrantes de la Comisión condujera la reunión. Asimismo, se indicó que alguien debía escribir las actas de reuniones. $Y$ en cuanto a esta cuestión, propusimos a sus integrantes que antes de comenzar las reuniones se leyera el acta anterior y, si era aprobada, se pasara al libro de Actas. Este ejercicio permitiría recordar los temas tratados con anterioridad y ver cómo habían ido evolucionando. Para poder sistematizar toda esta información de las reuniones también se diseñó un cuadro en donde quedaran registrados: los temas tratados, la situación particular que se presentara, qué resolvía la Comisión, qué tareas se debían realizar, quiénes serían sus responsables y cuál sería el plazo para cumplirlas. Finalmente, se realizaron dos talleres sobre toma de decisiones y resolución de problemas. En los mismos se reflexionó sobre situaciones problemáticas al interior de la Comisión Directiva y las formas para tratar de resolverlas adecuadamente. Igualmente, fue oportuno para fortalecer los vínculos interpersonales de sus integrantes. 
Estas modificaciones en la metodología de trabajo por parte del equipo del PEIS surgieron para superar las dificultades que se presentaron en la segunda etapa y conforman un intento para seguir fortaleciendo el proceso de autogestión de la Vecinal Villa del Parque y generar las condiciones necesarias para que establecer un diálogo de saberes y conocimientos entre los integrantes de la Comisión Directiva y los miembros del equipo.

Sin dudas, comenzar a coproducir un proyecto autogestionado en la Vecinal Villa del Parque implicó una práctica integral y una propuesta pedagógica nutrida desde el diálogo horizontal, no asimétrico, entre múltiples conocimientos. Así como una universidad de puertas abiertas "caminante y nómada" que "trille" muchos caminos, paisajes y pueblos" (Tommasino, 2009, p. 11), porque la propia autogestión es un proyecto y movimiento, es decir, no es un modelo acabado. Su estructura, organización y consolidación son y serán fruto del deseo, del pensamiento y de la acción de los miembros su Comisión Directiva, sin preconceptos ni imposiciones de modelos teóricos abstractos. La autogestión se transforma en un medio y un fin, en tanto la única forma de lograrla es mediante la ejecución de acciones autogestionarias, a través de la plena participación del individuo en el conjunto social, asumiendo en forma directa y colectiva la marcha de su grupo (Méndez y Vallota, 2006).

A manera de síntesis, el Cuadro 1 visualiza las etapas del proceso de implementación conforme a los actores involucrados, las dificultades encontradas y las modificaciones propuestas.

Cuadro 1: Síntesis de las etapas del proceso de implementación de los PEIS

\begin{tabular}{|c|c|c|c|}
\hline Etapas & $\begin{array}{l}\text { Primera etapa (2011-2013) } \\
\text { Acercamiento de la Asociación } \\
\text { Vecinal a la FCE. }\end{array}$ & $\begin{array}{l}\text { Segunda etapa (2014-2016) } \\
\text { 1er. PEIS: Desarrollo de las dos líneas de } \\
\text { acción de forma simultanea: } \\
\text { 1- Fortalecimiento institucional. } \\
\text { 2- Obra-escuela. }\end{array}$ & $\begin{array}{l}\text { Tercera etapa (2017-2019) } \\
\text { 2do. PEIS: centrándose la línea de } \\
\text { fortalecimiento institucional. }\end{array}$ \\
\hline $\begin{array}{l}\text { Actores } \\
\text { sociales }\end{array}$ & $\begin{array}{l}\text { Equipo de la FCE - integrantes } \\
\text { de la Comisión Directiva de la } \\
\text { Asociación Vecinal. }\end{array}$ & $\begin{array}{l}\text { Equipo de la FCE - integrantes de la } \\
\text { Comisión Directiva de la Asociación } \\
\text { Vecinal - integrantes de la Cooperativa de } \\
\text { trabajo Jóvenes de San Cayetano. }\end{array}$ & $\begin{array}{l}\text { Equipo de la FCE - integrantes } \\
\text { de la Comisión Directiva de la } \\
\text { Asociación Vecinal. }\end{array}$ \\
\hline $\begin{array}{l}\text { Dificultades } \\
\text { y obstáculos }\end{array}$ & $\begin{array}{l}\text { Necesidad de conocimiento } \\
\text { disciplinario y un profesional que } \\
\text { certifique el balance contable. }\end{array}$ & $\begin{array}{l}\text { - Los integrantes de la Comisión Directiva } \\
\text { no se apropian de una dinámica de tra- } \\
\text { bajo en equipo. } \\
\text { - Dificultades para diferenciar el rol } \\
\text { académico de los integrantes del equipo } \\
\text { del proyecto y el sentido de sus acciones. } \\
\text { - Dificultades de comunicación entre el } \\
\text { equipo del PEIS y la Comisión Directiva. } \\
\text { - Imposibilidad de consolidar el vínculo de } \\
\text { confianza con la Cooperativa de Trabajo. }\end{array}$ & $\begin{array}{l}\text { - Necesidad de organización de } \\
\text { las reuniones. } \\
\text { - Fortalecimiento del proceso de } \\
\text { toma de decisiones. } \\
\text { - Sistematización de las acciones } \\
\text { desarrolladas por los integrantes } \\
\text { de la Comisión Directiva. }\end{array}$ \\
\hline $\begin{array}{l}\text { Moficaciones } \\
\text { propuestas }\end{array}$ & $\begin{array}{l}\text { Establecimiento del vínculo } \\
\text { de confianza y proceso de } \\
\text { involucramiento en el contexto del } \\
\text { barrio. }\end{array}$ & $\begin{array}{l}\text { - Decisión de trabajar en el nivel micro- } \\
\text { económico. } \\
\text { - Reestructuración de la división de tareas } \\
\text { entre los integrantes del PEIS. } \\
\text { - Reuniones de Comisión Directiva como } \\
\text { canal de comunicación. } \\
\text { - Creación un correo electrónico institu- } \\
\text { cional del PEIS. }\end{array}$ & $\begin{array}{l}\text { - Consolidación del proceso de } \\
\text { autogestión. }\end{array}$ \\
\hline
\end{tabular}

Fuente: elaboración propia, 2020. 


\section{Conclusiones}

Comenzamos este trabajo realizando una revisión teórica sobre las implicancias de la propuesta de las Epistemologías del Sur, más específicamente sobre la ecología de saberes como herramienta para resignificar los vínculos entre los conocimientos de distintos actores que participaron de los proyectos de extensión presentados. Este enfoque permite el diálogo entre los conocimientos producidos en la universidad y los conocimientos y saberes cotidianos o populares, que enriquecen mutuamente a partir del reconocimiento de su diversidad. Asimismo, desde la ecología de saberes es posible comprender la autogestión no solo como un ejercicio en el cual se pone en juego un saber técnico especializado sino como un proceso de construcción a partir de un diálogo entre conocimientos y saberes.

Luego presentamos las distintas etapas por las que atravesaron los dos PEIS, que tuvieron como objetivo de contribuir al desarrollo institucional y a la capacidad de autogestión de la Comisión Directiva de la Asociación Vecinal Villa del Parque. La primera etapa surgió con el acercamiento de la Vecinal a la Universidad, la respuesta a una demanda puntual y el establecimiento de los primeros vínculos de confianza y conocimiento. Ello sirvió como andamiaje fundacional para dar continuidad al vínculo interinstitucional (Universidad-Vecinal), donde la construcción colectiva del diseño político de las acciones y la transparencia en el manejo de los presupuestos de los proyectos fueron determinantes para el proceso.

En la segunda etapa, por un lado, se asesoró y acompañó a la Comisión Directiva en cuestiones contables, administrativas, legales (confección del Balance Anual Contable y el Inventario, la apertura de una cuenta corriente en una entidad bancaria, la rendición de un subsidio del Ministerio de Desarrollo Social de la provincia para la construcción de un playón deportivo, y la reforma del Estatuto Social). Por otro lado, se trató de trabajar en el nivel mesoeconómico a partir de la articulación de la Vecinal con la Cooperativa de Trabajo Jóvenes de San Cayetano en la implementación de una estrategia de "obra-escuela".

A lo largo de esta segunda etapa se sucedió una serie de dificultades que obstaculizaron el proceso de autogestión y pusieron en riesgo el diálogo de saberes que se intentó construir. Identificamos dificultades internas de la Comisión Directiva vinculadas con la división de tareas y responsabilidad de cada integrante, como también en el proceso de toma de decisiones y resolución de problemas. $Y$ por otra parte se presentaron dificultades en la modalidad de comunicación entre la Comisión Directiva y el equipo del PEIS. Finalmente, fue problemático establecer un vínculo de confianza sólido entre la Asociación Vecinal y la Cooperativa Jóvenes de San Cayetano.

Considerando todas estas dificultades que surgieron en el proceso de intervención llevado a cabo, en la tercera etapa desde el equipo del PEIS se decidió realizar cambios en las estrategias de intervención, pasando del nivel mesoeconómico al microeconómico, focalizándose exclusivamente en la Comisión Directiva y siendo conscientes, a partir de las experiencias previas, de que primero se debe fortalecer el nivel micro como condición necesaria para desarrollar un proyecto a nivel meso.

Además, modificamos la metodología de trabajo utilizada con el propósito de mejorar el funcionamiento interno del equipo extensionista y agilizar la comunicación con la Comisión Directiva dotándola de una mayor formalidad. En esta tercera etapa se trató de generar herramientas que permitieran sistematizar la gestión de la Comisión Directiva para poder evaluar su evolución en el tiempo. Por último, se procuró fortalecer el proceso de toma de 
decisiones y resolución de problemas y las relaciones interpersonales entre los integrantes de la Comisión Directiva.

En el recorrido de este análisis consideramos que el principal desafío en la construcción del proceso autogestivo en la Comisión Directiva de la Vecinal Villa del Parque fue poder realizar constantemente una evaluación participativa junto con los actores sociales involucrados sobre las acciones implementadas de modo que fuera posible adaptarse a la dinámica cambiante del propio proceso. La evaluación del proceso autogestivo debe permitirnos tomar decisiones acertadas, confiables y fundamentadas acerca de cómo dar a nuestras acciones la direccionalidad deseable, basados en apreciaciones valorativas sobre lo que se realiza y los logros obtenidos.

Desde la dialéctica asumida y el proceso de reflexión concebido, el estudio genera nuevas preguntas y desafíos a nuestras propias prácticas. Las tensiones se renuevan y los sueños se reproducen. Y precisamente de esto se trata, de animarnos a problematizar a partir de nuestros propios resultados, con la firme convicción de que la resistencia es parte del propio proceso de ser promotores de la ESS. En este sentido, este trabajo trató de mirar hacia atrás buscando aprender de lo hecho, de los errores y los aciertos, valorando los aspectos positivos y los negativos, para continuar recorriendo los caminos hacia la autogestión de la Comisión Directiva de la Vecinal Villa del Parque.

\section{Referencias bibliográficas}

Azerrad, M. R. (2009). La experiencia de integración de extensión, docencia e investigación en la Universidad Federal de San Carlos en la formación de equipos de trabajo vinculados a la Economía Solidaria. III Congreso Nacional de Extensión Universitaria. Universidad Nacional del Litoral.

Carballeda, A. J. (2008). Los cuerpos fragmentados. La intervención en lo social en los escenarios de la exclusión y el desempleo. Paidós.

Coraggio, José L. (2016). La Economía Social y Solidaria (ESS): Niveles y alcances de acción de sus actores. http://www.economiasolidaria.org/documentos/la_economia_social_y_solidaria_ess_niveles_y_alcances_de_ accion_de_sus_actores_jlcoraggio

Coraggio, J. L. y Sabaté, A. F. (2010). Emprendimientos socioeconómicos asociativos: su vulnerabilidad y sostenibilidad. Universidad Nacional de General Sarmiento.

Méndez, N. y Vallota, A. (2006). Una perspectiva anarquista de la autogestión. Revista venezolana de economía y ciencias sociales, 12(1), 59-72.

López, Leandro S. (2005). La participación ciudadana a través del reclamo vecinal a principios del siglo XXI. Estudio comparado de tres formas de canalización de la demanda social en la Ciudad de Buenos Aires: Sociedades de Fomento, Sistema de Reclamos y Asambleas Barriales. Informe final del concurso: Poder y nuevas experiencias democráticas en América Latina y el Caribe. Programa Regional de Becas CLACSO.

Peixoto de Albuquerque, P. (2004). Autogestión. En Cattani, D., La Otra Economía (pp. 199-212). Altamira.

(2014). Elementos para repensar el concepto de autogestión. En Schujman, M. S.; Peixoto de Albuquerque, P.; Pereyra, K. C.; Tomatis, K. (Comps.). Economía Social y Solidaria: praxis, vivencias e intenciones. Ediciones del Revés.

Sousa Santos B. de y Rodríguez, C. (2007). Para ampliar el canon de la producción. Revista Otra economía, 1(1), 8-13. 
Sousa Santos B. de (2009). Una Epistemología del Sur. La reinvención del conocimiento y la emancipación social. 1ra. ed. CLACSO.

(2011). Epistemologías del Sur. Utopía y praxis latinoamericana, 16(54), 17-39.

(2018). Epistemologías del Sur - Epistemologías do Sul. 1ra ed. CLACSO Ediciones Coímbra: Centro de Estudos Sociais.

Tommasino, H. (2009). Generalización de las prácticas integrales. Los aportes de la extensión para su Implementación. III Congreso Nacional de Extensión Universitaria. Universidad Nacional del Litoral.

Ullberg de Baez, S. (2015). Desastre, memoria y economía solidaria. El caso de la ciudad de Santa Fe y sus inundaciones. +E: Revista de Extensión Universitaria, 5(5), 90-97. https://doi.org/10.14409/extension.v5i5.5147 